\title{
Editorial
}

\section{Energy Material Advances: From Fundamental Discoveries to Practical Applications}

\author{
Feng $W u^{1}$ and Jun Liu ${ }^{2}$ \\ ${ }^{1}$ Beijing Institute of Technology, China \\ ${ }^{2}$ University of Washington, USA \\ Correspondence should be addressed to Feng Wu; wufeng863@bit.edu.cn and Jun Liu; jliuuw1@uw.edu
}

Received 20 November 2020; Accepted 20 November 2020; Published 17 December 2020

Copyright (C) 2020 Feng Wu and Jun Liu. Exclusive Licensee Beijing Institute of Technology Press. Distributed under a Creative Commons Attribution License (CC BY 4.0).

In 2019, John B. Goodenough, M. Stanley Whittingham, and Akira Yoshino jointly received the Nobel Prize in Chemistry for their exceptional contributions to the development of lithium-ion batteries. Their pioneering and foundational work has enabled a new generation of powerful energy storage devices that have fundamentally transformed modern society. This award is not only a timely celebration of the scientists' outstanding achievements but also a testament to scientific discoveries in energy materials and technologies being one of the most remarkable discoveries made by mankind.

It is a grand challenge in today's world to supply sufficient and reliable energy to meet the requirements of a growing population and rapidly advancing technologies. The development and deployment of new materials play a central role in almost all new clean energy technologies, including high-efficient energy production, storage, conversion, and utilization. As demonstrated by the evolution of lithium-ion batteries, fundamental research in the discovery of new materials, increased understanding of material phenomena in multiscale and multicomponent systems, breakthroughs in material properties and performances, insight into how novel materials and discoveries can be integrated into functional devices, and efficient material synthesis, processing and manufacturing techniques constitute the cornerstones for successful development and commercialization of new energy technologies in the marketplace.

As a partner journal in the Science Partner Journal program, Energy Material Advances (EMA) was cofounded in 2020 by the Beijing Institute of Technology (BIT) and the American Association for the Advancement of Science
(AAAS). EMA is a unique journal; it is the first to cover the entire lifecycle of energy materials with a significant emphasis on cutting-edge, interdisciplinary research to accelerate the transformation from fundamental discoveries to practical applications. This journal is committed to be an effective platform in promoting international and interdisciplinary communication and collaboration on the latest scientific progress and breakthroughs of new energy materials and technologies.

EMA focuses on key scientific issues for the whole materials lifecycle: from basic material research to device integration, large scale application, end-of-life analysis, and recycling. The journal covers a range of subjects, from material discovery, chemistry, physics, informatics, machine learning, optoelectronics, to devices and vehicle integration, as well as relevant interdisciplinary research. EMA's scope includes not only fundamental studies of green energy materials, functional nanostructured materials, and high-energy density materials, but also research on the design, fabrication, and performance of high-energy secondary batteries, supercapacitors, fuel cells, solar cells, and other advanced energy devices. Furthermore, EMA is interested in progresses that link materials and device-level research and breakthroughs to major energy applications, including renewable energy, next-generation electronic communications, large-scale energy storage, clean energy vehicles, upgrading traditional energy, material recycling, and secondary applications.

The 21st century has seen an explosion of materials research efforts and reports on material discoveries, innovations, and potential applications. The world is entering a new era of integrated energy and information ecosystems 
that are highly interconnected. Under these circumstances, methods to rapidly integrate radical, disruptive innovations, and emerging energy technologies with existing systems present a formidable challenge for the scientific community. Currently, there is also a significant gap among different research disciplines and between the research community and industry. We earnestly hope that EMA will address these gaps and challenges, and provide a global platform for researchers, scientists, and engineers to exchange new ideas and share the latest knowledge and breakthroughs. We also hope EMA will be a launching point for discussions on innovative research and strategies to overcome the barriers between science, engineering, and different research and development communities.

The publications in EMA will be presented in the form of research articles, reviews, short communications, perspectives, and editorials. EMA will publish high-quality original research results, as well as critical reviews and analysis on the latest developments in energy materials, all from comprehensive and multifaceted perspectives. EMA will establish an efficient and rigorous peer review process. Manuscripts submitted to EMA will be reviewed in a timely manner by well-established experts and scholars in the relevant fields.

We eagerly welcome opinions, suggestions, and critiques from the international community to help EMA become not only a first-class journal in the energy field but also a valuable tool to accelerate scientific research and discoveries. We also thank our outstanding editorial team members for their great effort in launching this important journal. We sincerely hope that with the joint efforts of all authors, readers, reviewers, and editorial board members, EMA will make a unique contribution to the advancement of energy materials research and accelerate the transformation of scientific knowledge into practical applications.

\section{Conflicts of Interest}

The authors declare that there is no conflict of interest regarding the publication of this article. 